\title{
Breaking the Femtosecond Barrier in Multi-Kilometer Timing Synchronization Systems
}

\author{
Ming Xin, Member, IEEE, Kemal Şafak, Michael Y. Peng, Aram Kalaydzhyan, Patrick T. Callahan, Member, IEEE, \\ Wenting Wang, Oliver D. Mücke, and Franz X. Kärtner, Fellow, IEEE
}

(Invited Paper)

\begin{abstract}
To observe electronic dynamics in atoms, molecules, and condensed matter taking place on an attosecond time scale, next-generation photon science facilities like X-ray free-electron lasers and intense laser beamlines require system-wide attosecondlevel synchronization of dozens of optical and microwave signals up to kilometer distances. Here, we present for the first time a timing synchronization system that can meet the strict timing requirements of such large-scale facilities. We discuss some key enabling technologies including master-laser jitter characterization, local timing synchronization, new designs of attosecond-precision timing/phase detectors, and analyze fundamental noise contributions in nonlinear pulse propagation in fiber links. Finally, a complete 4.7-km laser-microwave network with 950 -as timing jitter is realized over tens of hours of continuous operation.
\end{abstract}

Index Terms-Optical synchronization techniques, attosecond photonics, large-scale timing distribution, laser-microwave network, free-electron lasers.

Manuscript received August 13, 2016; revised September 26, 2016 and September 27, 2016; accepted September 27, 2016. This work was supported by the European Research Council under the European Union's Seventh Framework Program (FP/2007-2013)/ERC Grant Agreement no. 609920 and the Cluster of Excellence: The Hamburg Centre for Ultrafast Imaging - Structure, Dynamics, and Control of Matter at the Atomic Scale of the Deutsche Forschungsgemeinschaft.

M. Xin is with the Center for Free-Electron Laser Science, Deutsches Elektronen-Synchrotron, Hamburg 22607, Germany, and also with the Research Laboratory of Electronics, Massachusetts Institute of Technology, Cambridge, MA 02139 USA (e-mail: ming.xin@cfel.de).

K. Şafak is with the Center for Free-Electron Laser Science, Deutsches Elektronen-Synchrotron, Hamburg 22607, Germany, and also with the Department of Physics and Hamburg Center for Ultrafast Imaging, University of Hamburg, Hamburg 22761, Germany (e-mail: kemal.shafak@cfel.de).

M. Y. Peng and P. T. Callahan are with the Research Laboratory of Electronics, Massachusetts Institute of Technology, Cambridge, MA 02139 USA (e-mail: mypeng@alum.mit.edu; pcallahn@mit.edu).

A. Kalaydzhyan and W. Wang are with the Center for Free-Electron Laser Science, Deutsches Elektronen-Synchrotron, Hamburg 22607, Germany (e-mail: aram.kalaydzhyan@cfel.de; wenting.wang@cfel.de).

O. D. Mücke is with the Center for Free-Electron Laser Science, Deutsches Elektronen-Synchrotron, Hamburg 22607, Germany, and also with the Hamburg Center for Ultrafast Imaging, University of Hamburg, Hamburg 22761, Germany (e-mail: oliver.muecke@cfel.de).

F. X. Kärtner is with the Center for Free-Electron Laser Science, Deutsches Elektronen-Synchrotron, Hamburg 22607, Germany, with the Research Laboratory of Electronics, Massachusetts Institute of Technology, Cambridge, MA 02139 USA, and also with the Department of Physics and Hamburg Center for Ultrafast Imaging, University of Hamburg, Hamburg 22761, Germany (e-mail: franz.kaertner@cfel.de).

Color versions of one or more of the figures in this paper are available online at http://ieeexplore.ieee.org

Digital Object Identifier 10.1109/JSTQE.2016.2614676

\section{INTRODUCTION}

S YNCHRONIZATION is an integral part of our world as it is widely used in many aspects of our daily life such as congestion resolution in intelligent transportation systems [1], time codes in film making [2], flash sync in digital cameras [3], MIDI clock in electronic music [4], and data packet switching in optical communications [5]. Timing precision of each synchronization technique ranges from several seconds to a few picoseconds, and maintaining this precision is a prerequisite to make our world more harmonious and more beautiful. With the ever increasing demands in science and technology, higher timing precision at the femtosecond (fs) and even attosecond (as) level has recently become desirable in large-scale networks for many advanced applications.

The first example is the telescope arrays employed in astronomy [6]-[10]. In order to obtain the surface details of distant astronomical objects, especially to explore exoplanets in the habitable zone, ultrahigh angular resolution such as $10^{-6}$ arcsecond (corresponding to $50 \mathrm{~km}$ spatial resolution at 1-lightyear distance) for cosmic imaging will be needed in the near future. To achieve this goal, the telescope array needs to work at terahertz or even optical wavelength [10] with hundreds-of-km scale. Then fs/as-precision synchronization among these telescopes are required to perform an accurate phase correlation of the ultrafast detected signals.

Gravitational waves have been directly detected by the Laser Interferometer Gravitational-Wave Observatory (LIGO) recently [11], [12] after a one-century search. However, developing new methods to make the detection more efficient is still necessary. An atomic-clock-based detector [13], [14] is one candidate, which is sensitive to the relative velocities of the reference masses, rather than the relative distance as in LIGO. In this scheme, a synchronized clock network with attosecond or even sub-attosecond precision is required, which will greatly increase the detection possibility and accelerate the development of gravitational-wave astronomy, if it can be realized. However, the required level of precision is an enormous challenge for current synchronization techniques.

Besides these applications discussed above, attosecondprecision synchronization is most urgently required in freeelectron lasers (FELs). Recently, several X-ray FELs (XFELs), such as the European XFEL [15] in Hamburg, FERMI [16] in Italy, SwissFEL in Switzerland and Linac Coherent Light 


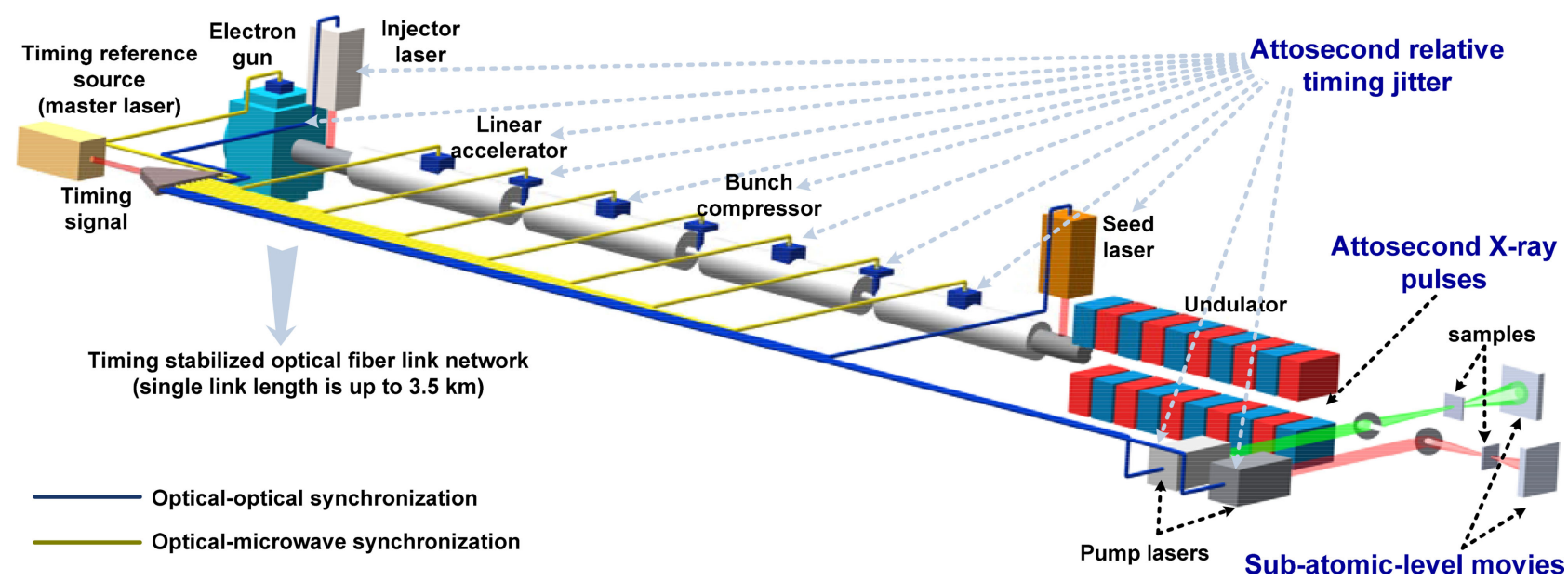

Fig. 1. Timing synchronization system in an attosecond XFEL [19].

Source (LCLS) [17] and LCLS II [18] in Stanford are built or being built worldwide with lengths ranging from a few hundred meters to several kilometers. All these facilities aim to generate attosecond X-ray pulses [20] with unprecedented brightness to film chemical and physical reactions with sub-atomic-level spatio-temporal resolution [21], [22]. Significant progress in the spectroscopy of attosecond-level molecular dynamics, including the time-domain observation of intramolecular charge transfer [23] and the discovery of ultrafast Auger processes altering the chemistry of matter on an attosecond time scale [24], [25], has been made in the past few years. However, the current XFELs are still not using their full potential for sub-atomic-level movie applications due to the lack of an attosecond-precision timing control to avoid blurring of the individual pictures in time. As illustrated in Fig. 1, in order to generate attosecond X-ray pulses and perform attosecond-precision pump-probe experiments, all the optical/microwave sub-sources inside an XFEL, including the electron gun, injector laser, microwave references of the linear accelerator and bunch compressor, seed laser and pump lasers at the end station, need to be synchronized simultaneously with attosecond relative timing jitter. Therefore, a multi-km attosecond-precision synchronization technique is imperative to unleash the full potential of these billion-dollar photon science facilities.

Generally, a synchronization system consists of a timing reference providing extremely stable timing signals, a target signal that needs to be synchronized, a timing detector that can measure the timing difference between the target signal and the reference, and a control box to lock the timing of the target to that of the reference. If the target device is far away from the reference, a timing link is also necessary to deliver the timing signal from the reference to the target. Without exception, the attosecond-precision synchronization technique also requires these key elements.

The timing reference source in attosecond synchronization can be an atomic clock [26], [27], a continuous-wave (CW) laser [28], [29] or a mode-locked laser [30], [31]. The state-of-theart technique in each solution can provide an attosecond-jitterequivalent instability for $1 \mathrm{~s}$ observation time. In contrast to the other two solutions, the mode-locked laser can simultaneously provide ultralow-noise optical and microwave signals, and the ultrashort optical pulses in the time domain can be directly used as time markers for precise timing measurements. So it is an ideal timing source for synchronization applications such as telescope arrays and XFELs, where the target devices are mode-locked lasers and microwave sources.

Another advantage of using mode-locked lasers is that it can provide very high sensitivity during timing detection, thanks to its high pulse peak power. For example, we have developed balanced optical cross-correlators (BOCs) [32], [33] and balanced optical-microwave phase detectors (BOMPDs) [34], [35] for optical-optical and optical-microwave timing detection, respectively. Both of them can achieve attosecond precision and $>1$-ps dynamic range at the same time. In contrast, it is difficult to build comparable timing detectors with atomic clocks or $\mathrm{CW}$ laser sources, due to their single-frequency property.

Usually the control box can be realized by a feedback loop, which needs to be carefully designed to avoid any feedbackinduced noise. For remote synchronization, the timing link can be either optical fiber [36]-[41] or free space [42], [43]. Freespace-based links are limited by the ground buildings and also suffer from atmospheric turbulences. But it may be suitable for space applications such as gravitational-wave detection. Optical-fiber-based timing links, on the other hand, are very flexible for installation and can be easily fitted into telescope arrays and XFELs.

In this work, we focus on the XFEL application, since it possesses currently the most urgent timing challenge. But the techniques we present here can also easily be adapted to other applications in the future. Based on the discussions above, the best synchronization solution for XFELs, as depicted in Fig. 1, should use a mode-locked laser (master laser) as the timing reference, and optical fiber links to distribute the timing signals to different remote laser/microwave sources. We have been working on this approach over the past decade [38] and already breached the 10-fs precision level [44]-[46], which is more than an order of magnitude better than the best results achieved with traditional microwave signal distribution schemes [47], [48]. In order to meet the strict timing requirements of XFELs, a novel attosecond-precision timing synchronization system is 


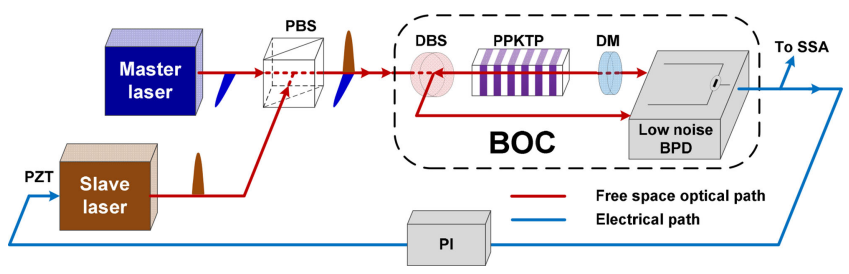

Fig. 2. Master-laser jitter characterization setup (PBS, polarization beam splitter; DBS, dichroic beam splitter; DM, dichroic mirror; PPKTP, periodicallypoled $\mathrm{KTiOPO}_{4}$; PI, proportional-integral controller; SSA, signal source analyzer).

developed based upon our previous work, and presented in the following of this paper. In Section II, we first show the timing jitter characterization of the master laser. Then local synchronization results and new BOMPD/BOC designs are reported in Section III. Link stabilization, link-induced timing jitter and the link feedback model are discussed in Section IV. Finally, a complete $4.7-\mathrm{km}$ laser-microwave network with attosecond precision is presented in Section $\mathrm{V}$ before we conclude in Section VI.

\section{MASTER-LASER JitTER CHARACTERIZATION}

Since the master laser in Fig. 1 serves as timing reference of all the optical/microwave sub-sources, it must exhibit attosecondlevel timing jitter, which needs to be accurately characterized. In conventional jitter characterization of mode-locked lasers, the laser output is first detected by a photodetector and then the phase noise is converted into an amplitude change by a mixer [49] for baseband power spectrum measurement. This scheme, which is adopted by state-of-the-art signal source analyzers (SSAs), suffers from amplitude-to-phase (AM-PM) noise conversion during photodetection [50], [51]. Here we use a different characterization method based on BOC [52], [53], which is intrinsically immune to AM-PM noise by directly converting the timing difference of two optical pulses into a voltage signal. The BOC characterization has achieved extremely low noise floors down to $10^{-12} \mathrm{fs}^{2} / \mathrm{Hz}$ for offset frequencies up to the Nyquist frequency of mode-locked lasers [31], [54].

The master-laser jitter characterization setup is given in Fig. 2. The output of two identical lasers (master and slave, with $216.667 \mathrm{MHz}$ repetition rate, $50 \mathrm{~mW}$ average power, 170 fs pulse width and $1553 \mathrm{~nm}$ center wavelength) were combined by a polarization beam splitter (PBS) and launched into a BOC, which consists of a single 4-mm-long periodicallypoled $\mathrm{KTiOPO}_{4}$ (PPKTP) crystal operating in a double-pass configuration with appropriate dichroic beam splitter and mirror (DBS, DM) and a low-noise balanced photodetector (BPD). The BOC output was fed back to the slave laser's PZT (with a sensitivity of $17.4 \mathrm{~Hz} / \mathrm{V}$ ) through a proportional-integral (PI) controller so that the two lasers' repetition rates were locked to each other. Finally, the BOC output was sent to an SSA for jitter characterization.

Based on the BOC locking model in [45], [46], [55], the timing jitter spectral density at the output of the BOC satisfies

$$
\overline{J_{O}^{2}}=2 C_{M} \overline{J_{M}^{2}}+C_{N} \overline{J_{N}^{2}}+\overline{J_{B}^{2}}
$$

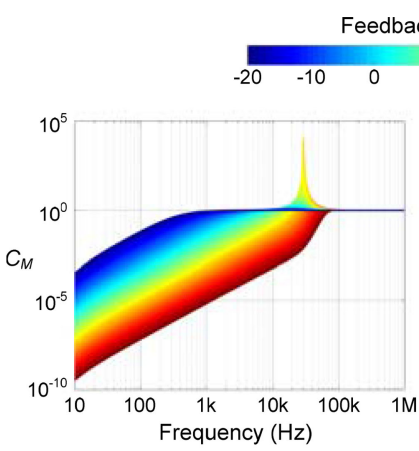

(a)

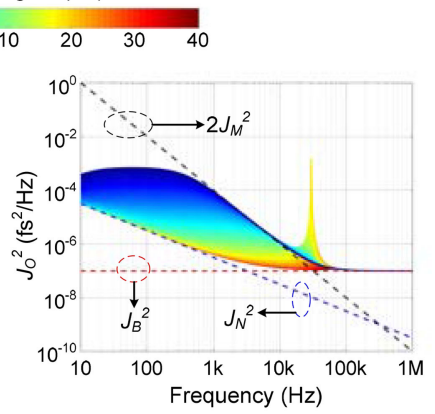

(b)
Fig. 3. (a) Coefficient $C_{M}$ at different feedback gain values; (b) Simulated BOC output jitter spectral density at different feedback gain values. The peaks around $30 \mathrm{kHz}$ in (a) and (b) originate from the PZT resonance of the slave laser.

where $C_{M}=|1 /(1+\boldsymbol{H})|^{2}, C_{N}=|\boldsymbol{H} /(1+\boldsymbol{H})|^{2}, \boldsymbol{H}$ is the total complex transfer function of the feedback loop, $J_{M}$ is the free-running jitter of the master laser, the factor 2 stems from the assumption that the master and slave lasers have identical but uncorrelated jitter, $J_{N}$ is the electronic noise from the feedback loop, and $J_{B}$ is the BPD noise directly coupled into the SSA. In order to use the measured value $J_{O}$ to estimate $J_{M}$, we should let $\boldsymbol{H} \approx 0$. Then $C_{M} \approx 1, C_{N} \approx 0$, an upper limit estimation of $J_{M}$ can be given

$$
\overline{J_{M}^{2}} \approx \frac{1}{2} \overline{J_{O}^{2}}-\frac{1}{2} C_{N} \overline{J_{N}^{2}}-\frac{1}{2} \overline{J_{B}^{2}} \leq \frac{1}{2} \overline{J_{O}^{2}}
$$

Using the feedback parameters from the experiments, we calculate $C_{M}$ at different feedback gain values, as shown Fig. 3(a). When the gain is at $-20 \mathrm{~dB}, C_{M}$ is almost 1 above $1 \mathrm{kHz}$. Furthermore, in Fig. 3(b), we assume that $J_{M}^{2}$ has a $1 / f^{2}$ slope (black dashed line), $J_{N}^{2}$ has a $1 / f$ slope (blue dashed line) and $J_{B}^{2}$ is a constant (red dashed line), so that we can easily analyze how each noise source affects $J_{O}$. It can be seen that as feedback gain increases, $J_{O}$ approaches $J_{N}$ from $10 \mathrm{~Hz}$ to $1 \mathrm{kHz}$, and above $50 \mathrm{kHz}, J_{O}$ is always limited by $J_{B}$ since $J_{B}$ dominates the noise sources. While below $50 \mathrm{kHz}$, more and more low-frequency components of $J_{O}^{2}$ coincide with $2 J_{M}^{2}$ when gain decreases. So in terms of measurement, we can decrease the feedback gain as much as possible (e.g., $-20 \mathrm{~dB}$ ), then we can obtain the accurate master-laser jitter between $1 \mathrm{kHz}$ and $20 \mathrm{kHz}$ and an upper-limit estimate above this frequency range.

The master laser measurement results are displayed in Fig. 4. The top panel shows the jitter spectral density at different feedback gains. As predicted by the simulations, the jitter spectrum is limited by the detector noise floor (grey curve) above $30 \mathrm{kHz}$. Between $1 \mathrm{kHz}$ and $30 \mathrm{kHz}$, as the gain decreases, the spectrum approaches the real laser jitter. We choose the lowest gain value (about $-15 \mathrm{~dB}$ ) at which the locking is still stable enough to perform a measurement, and obtain 330 as integrated timing jitter from $1 \mathrm{kHz}$ to $1 \mathrm{MHz}$, as shown in the bottom panel of Fig. 4 . This value gives a very good upper-limit estimate of the master laser's jitter. So this laser is definitely capable of providing the reference in an attosecond-precision timing synchronization system. 


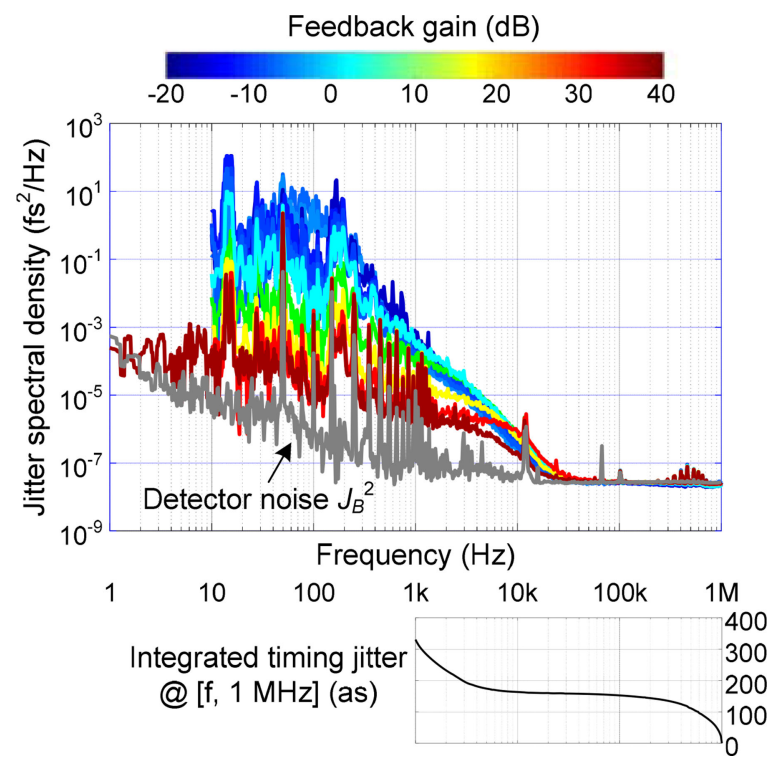

Fig. 4. Measured master-laser jitter spectrum and corresponding integrated timing jitter.

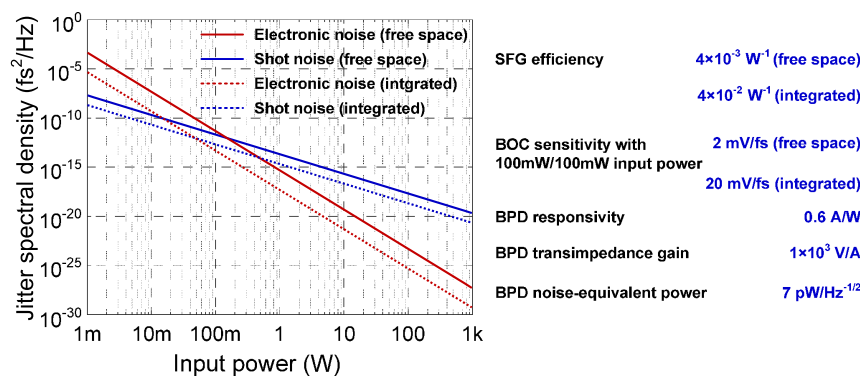

Fig. 5. Noise floor of BOC at different input average power levels (SFG, sum-frequency generation; BPD, balanced photodetector).

In practice, the timing precision of a $\mathrm{BOC}$ is either limited by the detector electronic noise or the optical shot noise. Using some typical experimental parameters, as listed in Fig. 5, this limitation can be estimated. We assume that the two input optical pulse trains of a BOC have the same average power, which are given on the horizontal axis of Fig. 5, then the BOC noise floor introduced by shot noise and electronic noise are calculated, respectively. The free-space-coupled BOC (as used in Fig. 2) is shot-noise limited above $200 \mathrm{~mW}$ input optical power otherwise it is limited by the electronic noise (blue/red solid curves in Fig. 5). With $1 \mathrm{~kW}$ input power, we can even achieve one-zeptosecond timing precision at $100 \mathrm{MHz}$ bandwidth. By replacing the free-space BOC with an integrated version [56], [57], the sum-frequency generation (SFG) efficiency can be increased by at least ten times, as a result the shot/electronic noise floor can be further decreased by $10 / 20 \mathrm{~dB}$ (blue/red dotted curves in Fig. 5).

\section{LOCAL SYNCHRONIZATION}

\section{A. Local Optical-Optical Synchronization}

After characterizing the master-laser jitter, it is necessary to test its local synchronization performance before installing it

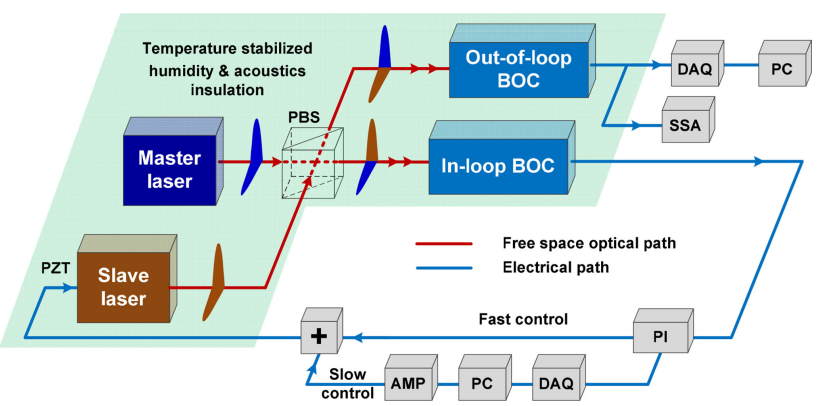

Fig. 6. Local optical-optical synchronization (DAQ, data acquisition card; PC, computer, AMP, voltage amplifier; + , voltage summer).

in a distributed network. The local optical-optical synchronization experimental setup is shown in Fig. 6. Similar to that in laser characterization, the repetition rates of the slave and master lasers were first locked together with an in-loop BOC, then another out-of-loop BOC was used to evaluate the jitter performance after synchronization. Both of the two BOCs have the same structure as that shown in Fig. 2. In the feedback loop, the output of the in-loop BOC was first filtered by a PI controller. Then the PI output was separated into two paths: the first path was directly sent to the slave laser's PZT without amplification to compensate fast jitter above $10 \mathrm{~Hz}$; the second path was sampled by a data acquisition (DAQ) card, analyzed by a Labview program to generate a DC voltage to compensate slow jitter below $10 \mathrm{~Hz}$, and a voltage amplifier was used to extend the compensation range. This feedback design can effectively optimize the locking bandwidth and compensation range simultaneously.

To minimize the thermally-induced timing fluctuations, the two lasers, two BOCs and other free-space optics were mounted on a temperature-stabilized breadboard with a Super-Invar surface sheet. With temperature fluctuations controlled below $\pm 0.05 \mathrm{~K}$, the effective timing instability of free-space beam paths due to thermal expansion is only $\pm 1 \mathrm{as} / \mathrm{cm}$. Lead foam was placed beneath the setup to damp table vibrations. A twolayer enclosure with acoustic heavy foil for the inner layer and high-density polyethylene (HDPE) for the outer layer was built to provide acoustic isolation for all optical components. A humidity insulation material covering the outer enclosure was also used to protect the setup from environmental humidity changes.

Fig 7(a) shows the out-of-loop jitter spectrum from $1 \mathrm{~Hz}$ to $1 \mathrm{MHz}$. The total integrated jitter over this frequency range is only 450 as. A long-term drift measurement was taken and the peak-to-peak drift in 10 hours is 400 as, which gives a root-mean-square (RMS) drift of 71 as (see Fig. 7(b)). The Fourier transform of the drift data is also calculated in Fig. 7(c). The integrated drift from $200 \mu \mathrm{Hz}$ to $1 \mathrm{~Hz}$ is only 50 as. These results indicate that optical synchronization using BOC can easily achieve attosecond precision. Furthermore, they also provide a precision limit that we can approach in the remote timing synchronization.

\section{B. Free-Space-Coupled BOMPD}

The optical-to-microwave synchronization can be realized by the BOMPD technique, and local synchronization experiments 


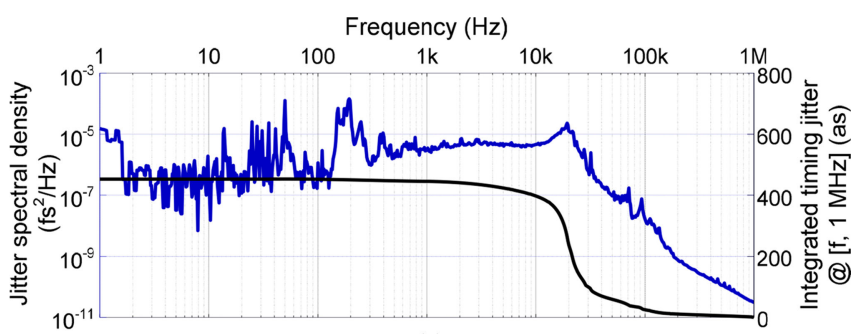

(a)

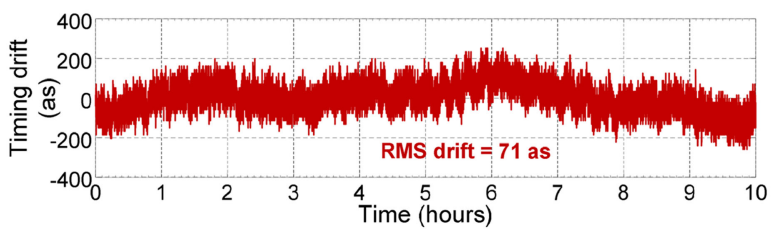

(b)

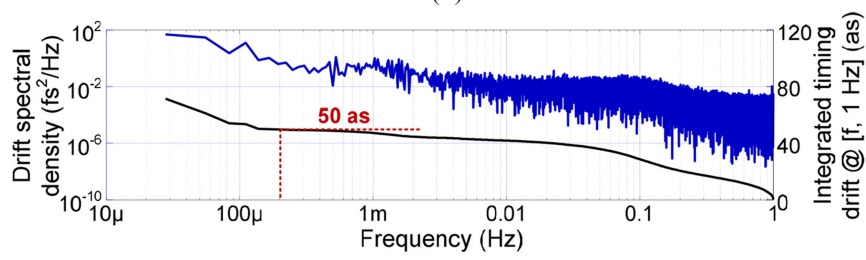

(c)

Fig. 7. Local optical-optical synchronization measurement results. (a) Outof-loop jitter spectrum; (b) long-term timing drift (sampling rate: $2 \mathrm{~Hz}$ ); (c) timing drift spectrum.

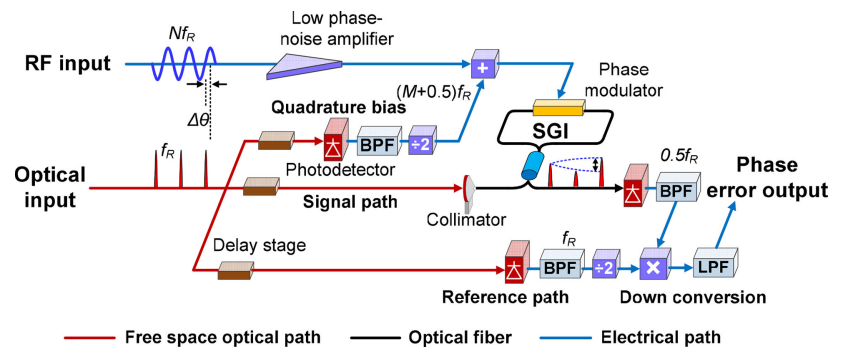

Fig. 8. Architecture of free-space-coupled BOMPD [19] $\left(f_{R}\right.$, the repetition rate of the optical input signal; $\Delta \theta$, the relative phase difference between the $\mathrm{RF}$ and optical input signals; SGI, Sagnac interferometer, BPF, bandpass filter; $\mathrm{LPF}$, lowpass filter; $\div 2$, frequency divider; + , voltage summer; $\times$, frequency mixer).

have been demonstrated in our previous works [35], [58]. However, the non-optimized design of BOMPD limits its local synchronization precision to the femtosecond level, which can only become worse in the remote case. In order to meet the km-scale attosecond-precision requirement in XFEL microwave synchronization, we have developed a new BOMPD configuration, free-space-coupled BOMPD (FSC-BOMPD) [19], as shown in Fig. 8.

The principle of this FSC-BOMPD can be explained as follows. An optical pulse train with a repetition rate $f_{R}$ from a mode-locked laser is launched into the FSC-BOMPD through the optical input port. The optical power is then separated into three paths: signal, bias and reference, with free-space components, such as PBS, half-wave plates and quarter-wave plates. Compared with the fiber-coupled approach [35], [58], this effi- ciently reduces the long-term drifts caused by the environment. Each path contains a free-space delay stage that enables precise phase tuning without backlash, microwave reflection and loss when compared with RF phase shifters. In the signal path, the optical signal is coupled into a Sagnac interferometer (SGI) through a collimator. If the pulse is sufficiently short, the optical pulse train power at the SGI input can be approximated by

$$
P_{i n}(t)=P_{a} T_{R} \sum_{n=-\infty}^{\infty}\left(1+\Delta_{R I N}(t)\right) \delta\left(t-n T_{R}-\Delta_{J}(t)\right)
$$

where $\delta(t)$ is the Dirac delta function, $T_{R}=1 / f_{R}$ is the period of the pulse train, $P_{a}, \Delta_{R I N}(t)$ and $\Delta_{J}(t)$ are the average power, power fluctuations and timing jitter of the pulse train, respectively.

In the bias path, a self-referenced signal $(M+0.5) f_{R}$ is derived from the optical pulse train after a photodetector (PD), bandpass filter (BPF) and a frequency divider, and sent to the phase modulator of the SGI together with the RF input signal at frequency $f_{0} \approx N f_{R}$. Usually $M$ is a very large number, the multi-GHz modulation in the phase modulator can ensure unidirectional phase modulation, which eliminates the repetition-rate dependence of the SGI, thus improving its robustness and longterm stability. The driving signal of the phase modulator can be written as

$$
\begin{aligned}
\varphi(t)=V_{0} \sin \left[2 \pi f_{0}\left(t+\Delta t_{0}\right)\right] \\
+V_{b} \sin \left[2 \pi(M+0.5) f_{R}\left(t+\Delta t_{b}\right)+\Delta \phi\right]
\end{aligned}
$$

where $V_{0}$ and $\Delta t_{0}$ are the amplitude and timing jitter of the RF input signal, respectively. $V_{b}$ is the amplitude of the RF signal from the bias path. $\Delta \phi$ and $\Delta t_{b}$ are the fixed relative phase and relative timing jitter between the pulse train and the RF bias signal, respectively.

Under quadrature bias condition, we have

$$
V_{b} \sin (\Delta \phi)=\pi / 2
$$

We suppose the frequency of the RF and optical input signals are locked with each other by the FSC-BOMPD, then $f_{0}=$ $N f_{R}$. Using (3)-(5), the output optical power after circulating in the SGI can be expressed as

$$
\begin{aligned}
P(t)= & (1-\alpha) P_{i n}(t) \sin ^{2}(\varphi / 2) \\
= & \frac{1-\alpha}{2} P_{a} T_{R} \sum_{n=-\infty}^{\infty}\left[1+V_{b}\left(-\frac{\theta_{b}^{2}}{2} \sin \Delta \phi+\theta_{b} \cos \Delta \phi\right)\right] \\
& \times\left(1+\Delta_{R I N}\right) \delta\left(t-n T_{R}\right)+\frac{1-\alpha}{2} P_{a} T_{R} \sum_{n=-\infty}^{\infty} V_{0} \theta_{e} \\
& \times\left(1+\Delta_{R I N}\right) \delta\left(t-n T_{R}\right) e^{j \pi f_{R} t}
\end{aligned}
$$

where $\alpha$ is the loss of the SGI, $\theta_{e}=2 \pi f_{0}\left(\Delta t_{0}+\Delta_{J}\right)$ is the relative phase error between the SGI and RF input signals, and $\theta_{b}=2 \pi(M+0.5) f_{R} \Delta t_{b}$ is the phase fluctuations of the bias path.

The SGI output is filtered by a BPF at $0.5 f_{R}$, so as to perform a frequency down-conversion with the signal in the reference 


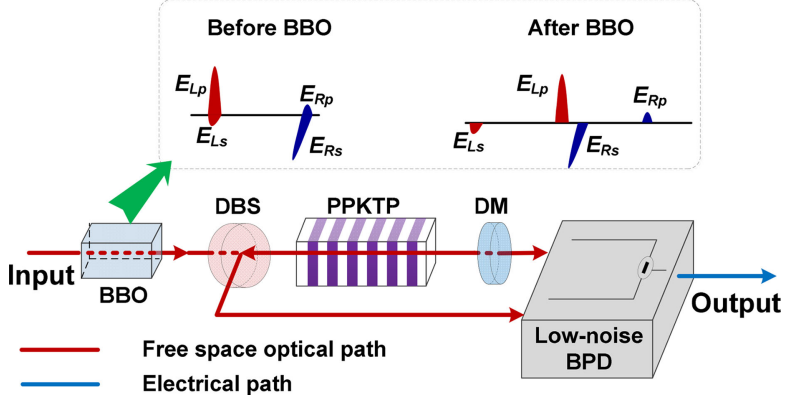

Fig. 9. Principle of polarization-noise-suppressed BOC [19] (PNS-BOC) (BBO, beta-barium borate crystal).

path. Since the $0.5 f_{R}$ frequency component only appears in the second term on the right-hand side of (6), the phase fluctuations $\theta_{b}$ from the bias path cannot affect this down-conversion process.

Finally, the error signal after down-conversion is

$$
V_{e}=\frac{C}{2}(1-\alpha) P_{a} T_{R} V_{r p} V_{0} \theta_{e}\left(1+\Delta_{R I N}\right) \cos \left(\pi f_{R} \Delta t_{r}\right)
$$

where $V_{r p}$ is the amplitude of the RF signal in the reference path, $\Delta t_{r}$ is the relative timing jitter between the reference and the SGI path, and $C$ is a constant coefficient related to the electronics during down-conversion. Since the down-conversion is performed as the lowest possible frequency $0.5 f_{R}$, the thermally induced phase changes in the reference path can be minimized. For example, if $f_{R}=216 \mathrm{MHz}$, a 3-mm length change from the reference path can only introduce $2 \times 10^{-5}$ relative change to $V_{e}$. Similarly, since usually $\Delta_{R I N} \ll 1$, in contrast to other BOMPDs [59], [60], $V_{e}$ is also insensitive to optical input power fluctuations. Thus, $V_{e}$ is mainly determined by $\theta_{e}$, the relative timing jitter between the RF and SGI input signals. The freespace optical paths before the SGI are well isolated from environmental changes, so that the FSC-BOMPD can accurately detect the timing jitter between the RF and optical input signals without introducing systematic errors.

\section{Polarization-Noise-Suppressed BOC}

In order to further improve the noise performance during timing detection, we also developed a new timing detector, polarization-noise-suppressed BOC (PNS-BOC) [19], as shown in Fig. 9. In a BOC, ideally the input pulses $\left(E_{L p}\right.$ and $\left.E_{R s}\right)$ are aligned along the two principal axes of the type-II PPKTP crystal for maximum SFG. Due to finite polarization extinction ratios in the optical elements upstream from the BOC, there will be undesired pulse components ( $E_{L s}$ and $E_{R p}$ ) projected along the undesired polarization axes. In the PNS-BOC, a linear material with large birefringence is placed before the BOC. This material adds a significant delay to the undesired components such that they do not overlap and interfere with the SFG process in the PPKTP crystal. In our setup, a BBO crystal is used to provide the required birefringence, whose cut angle is carefully selected to make sure that it cannot generate any nonlinear process.

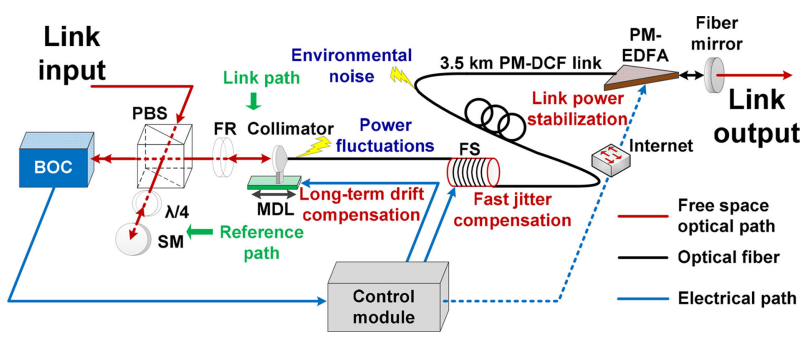

Fig. 10. General link stabilization setup [19] (SM, silver mirror; $\lambda / 4$, quarterwave plate; FR, $45^{\circ}$ Faraday rotator; MDL, motorized delay line; FS, fiber stretcher; $3.5-\mathrm{km}$ PM-DCF link, $3.5 \mathrm{~km}$ polarization-maintaining dispersioncompensated fiber link; PM-EDFA, polarization-maintaining erbium-doped fiber amplifier).

\section{LINK STABILIZATION}

\section{A. 3.5-km Timing Link Stabilization}

Timing link stabilization is crucial for remote timing synchronization, and may become the jitter-limiting factor if not carefully optimized. Fig. 10 shows a general link stabilization setup using polarization-maintaining (PM) fiber. The link input signal is first divided into reference and link path pulses. The reference path length is set as short as possible (e.g., $4 \mathrm{~cm}$ ) to minimize timing errors introduced by environmental noise. A fiber mirror is placed at the end of the link to partially reflect the link pulses' power back for stabilization. To ensure that the forward and backward link transmission accumulates the same amount of jitter, the link pulse must travel along the same polarization axis during round-trip propagation. Therefore, a $45^{\circ}$ Faraday rotator before the fiber link is necessary to introduce a $90^{\circ}$ round-trip polarization rotation to direct the reflected link pulse towards the BOC. In the control module, the output voltage of the PI controller is divided into two paths. The first path compensates for fast noise in the link and usually consists of a high-voltage amplifier that drives a PM fiber stretcher. The second path compensates for long-term environmental drift; a DAQ card is usually used to sample the timing error, and control commands can be sent to a motorized delay line (MDL) through a Labview program.

A $3.5-\mathrm{km}$ link stabilization experiment is demonstrated using the setup in Fig. 10. The output of the master laser used in Sections II and III was separated into two paths, the pulses in one path was sent to the link input port of Fig. 10, and the pulses of the other path were compared with the link output power with an out-of-loop BOC in free space, to evaluate the link stabilization performance. The out-of-loop jitter spectrum is given in Fig. 11(a), the total integrated jitter from $1 \mathrm{~Hz}$ to $1 \mathrm{MHz}$ is about 340 as, which are mainly contributed by the master laser's inherent jitter and the feedback loop. The link was continuously locked over 96 hours yielding an RMS drift of 570 as (see Fig. 11(b), red curve). We also performed another long-term measurement by replacing the out-of-loop free-space BOC with a fiber-coupled integrated one [57], and obtained 500 as RMS drift (see Fig. 11(b), black curve) over 84 hours. The two long-term measurements show similar magnitudes of drift over tens of hours, while the drift in a 1-hour period with a 


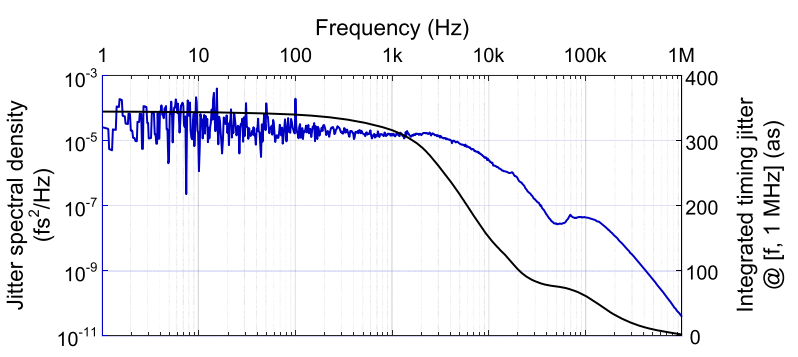

(a)

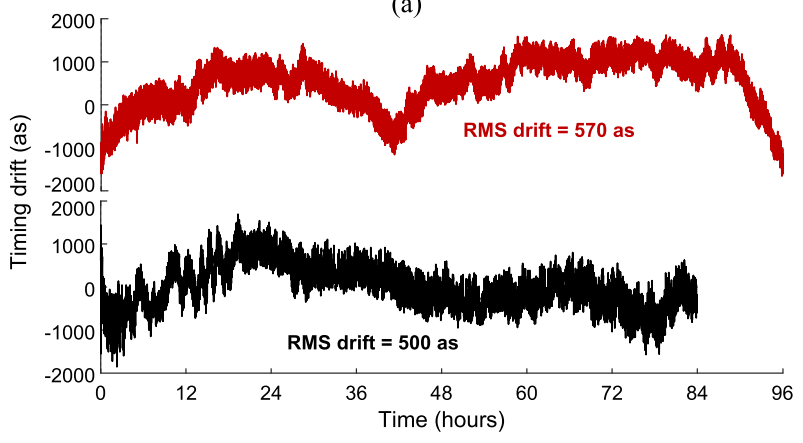

(b)

Fig. 11. 3.5-km timing link stabilization results. (a) Out-of-loop jitter spectrum; (b) two independent long-term timing drift measurements using a freespace BOC (red) and a fiber-coupled integrated BOC (black).

fiber-coupled BOC is a little worse because the fiber is more sensitive to environmental changes [46].

\section{B. Link-Induced Timing Drift and Jitter}

As indicated by Fig. 11(b), there are $\sim 2.5$-fs peak-to-peak timing drifts in the link stabilization, which cannot be eliminated by replacing the out-of-loop BOC. The noise source for the long-term drift must be targeted before we tackle any further optimization. In fact, in the timing stabilization setup of Fig. 10, long-range compensation is performed by a freespace MDL with long delay arms; e.g., a 10-cm range is required to correct for \pm 1.5 -K temperature change in a $3.5-\mathrm{km}$ link. Movement of the delay stage introduces inevitable beam misalignments that cause link power fluctuations. These fluctuations induce temporal shifts in the pulses' center-of-gravity (COG) through a composite effect of link residual second-order dispersion (SOD), third-order dispersion (TOD) and nonlinearity. Although a COG shift appears as a deterministic shift in the zero-crossing position of the in-loop BOC characteristic (i.e., the BOC output voltages with respect to the initial delay of the two input pulses), the link stabilization feedback will unknowingly track this shift and erroneously introduce it into the link path, causing a timing error at the link output.

To confirm this link-induced effect, we performed simulations using typical parameters from the experiments. Self-phase modulation, self-steepening and the Raman effect are considered in the link. Both the nonlinear Schrödinger equation [61] for the link transmission and the coupled field equations [62] for the SFG process in the BOC were solved using the split-step Fourier method with adaptive step length. The BOC characteristic was calculated for each round-trip link pulse against a new laser reference pulse. The timing offset of the zero-crossing po-

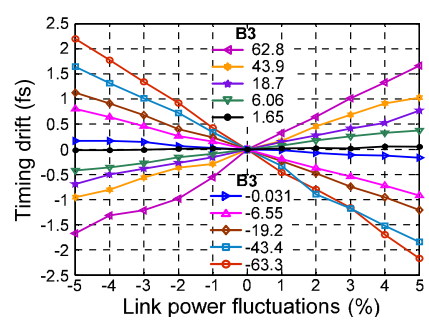

(a)

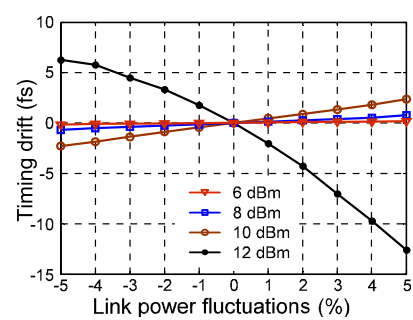

(b)
Fig. 12. Timing drift introduced by link power fluctuations (in (a), the input power is $+8 \mathrm{dBm}$ and $\mathrm{B} 2=-0.13$; in (b), $\mathrm{B} 2=-0.13$ and $\mathrm{B} 3=18.7$ ) [19].

sition in the BOC characteristic was identified as timing error. The parameters B2/B3 were used to represent the link residual SOD/TOD normalized by the SOD/TOD of 1-m standard PM 1550 fiber. Fig. 12(a) shows that residual TOD can induce up to 5 fs timing error for +8 - $\mathrm{dBm}$ link power with $\pm 5 \%$ fluctuations. Fig. 12(b) indicates that $+10-\mathrm{dBm}$ link power is the threshold before significant amplitude-to-timing conversion occurs due to severe nonlinear pulse distortions that may result in $4 \mathrm{fs}$ of timing error from $\pm 5 \%$ power fluctuations.

In our experiments, the timing links were constructed employing a section of standard PM 1550 fiber, followed by a section of PM dispersion-compensating fiber. Due to a little difference between the ratios of SOD to TOD coefficients in each fiber, when the SOD of a $3.5-\mathrm{km}$ link was completely eliminated, 26-m worth of TOD from PM 1550 would remain uncompensated. Based on Fig. 12(a), this residual TOD can introduce a drift of at least 2 fs for $\pm 5 \%$ link power fluctuations, which agrees with the results in Fig. 11(b). In order to eliminate these power-fluctuations-induced drifts, the link power must be stabilized. As shown in Fig. 10, this can be realized by sending the third feedback signal (dashed blue line) to the PM-EDFA through internet to adjust its pump current.

Besides the long-term drifts, the fast jitter of an optical pulse train can also be enhanced during fiber link transmission. We also developed a model to investigate this effect. In the model, the master equation of a fast-saturable-absorber mode-locked laser was solved using the fourth-order Runge-Kutta in the interaction picture (RK4IP) method [63]. Laser timing jitter was generated by adding amplified spontaneous emission (ASE) noise during each iteration of RK4IP, whose amount corresponds to the measured jitter of the master laser in Section II. The calculation was repeated for a train of laser pulses in the presence of pulse timing jitter and each pulse was solved by similar procedures in the link-induced drift simulation.

In Fig. 13, pulse center-frequency fluctuations are converted into timing jitter via residual SOD and TOD. This jitter contribution, often called Gordon-Haus jitter [64], can amount to 0.15 and 0.3 fs for uncompensated SOD equivalent to 2 and $3 \mathrm{~m}$ of standard PM fiber, respectively.

In Fig. 14, spontaneous emission noise is converted into timing jitter and its impact is further enhanced by link nonlinearities. The introduced jitter is bounded at $0.13 \mathrm{fs}$ for average power levels below $+12 \mathrm{dBm}$ (corresponding to a pulse peak power of $\mathrm{P}_{\text {peak }}=430 \mathrm{~W}$ ) but escalates to $1.4 \mathrm{fs}$ at $+14 \mathrm{dBm}$ $\left(\mathrm{P}_{\text {peak }}=682 \mathrm{~W}\right)$. This jitter needs to be carefully minimized 


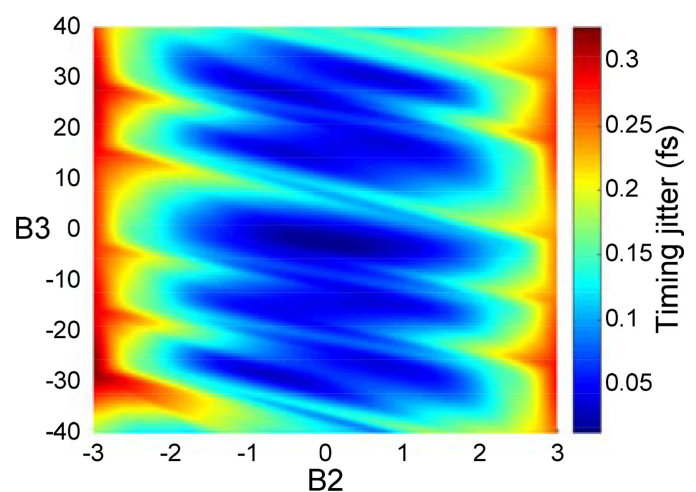

Fig. 13. Link-induced Gordon-Haus timing jitter for different B2/B3 values.

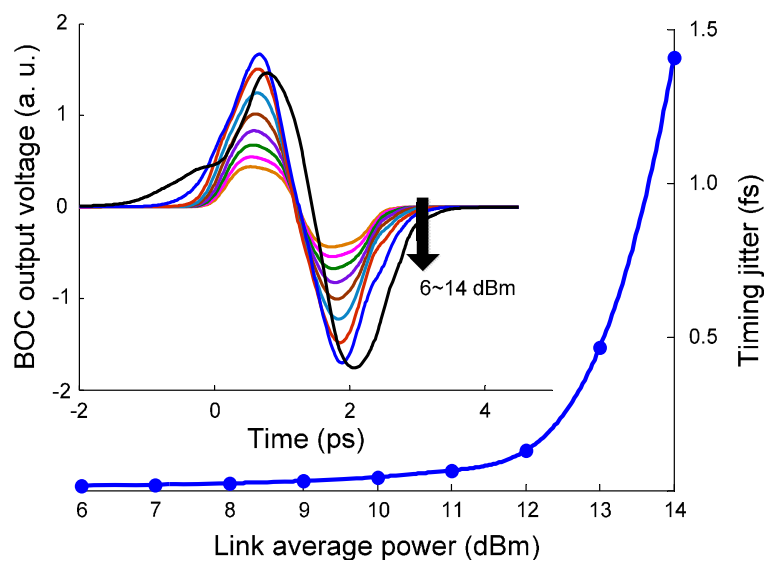

Fig. 14. Link-induced timing jitter due to nonlinear effects in the fiber [19].

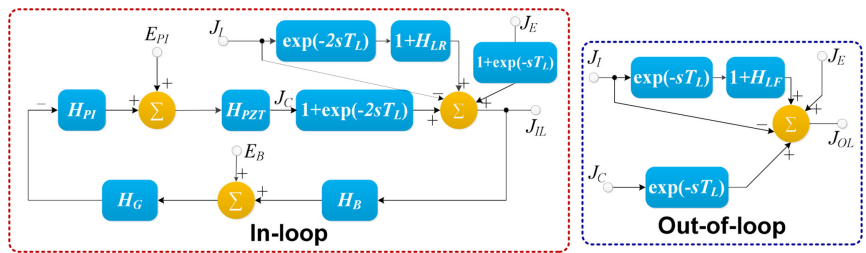

Fig. 15. Timing-link feedback model $\left(H_{B}, H_{G}, H_{P I}, H_{P Z T}\right.$, the transfer function of in-loop BOC, BPD transimpedance amplifier, PI controller and fiber stretcher; $T_{L}$, the single-trip link transmission time; $H_{L F} / H_{L R}$, the equivalent transfer functions of the link-induced timing jitter for forward/round-trip transmission; $E_{B}, E_{P I}$, the electronic noise of the BPD in the in-loop BOC and the PI controller; $J_{I}$, master laser's inherent jitter; $J_{E}$, integrated environmental jitter imposed on the link for single-trip link transmission; $J_{C}$, equivalent timing delay generated by the control loop for compensation; $J_{I L} / J_{O L}$, detected timing jitter by the in-loop/out-of-loop BOC); $s=j \omega$, complex frequency.

in practice because it easily reaches the fs-level before a visible distortion of the BOC characteristic can be observed, as shown in the inset of Fig. 14.

\section{Link Feedback Model}

In order to investigate how link-induced noise can affect the performance of the timing distribution system, we also developed a feedback model for the timing link. A flow diagram of this model is shown in Fig. 15.

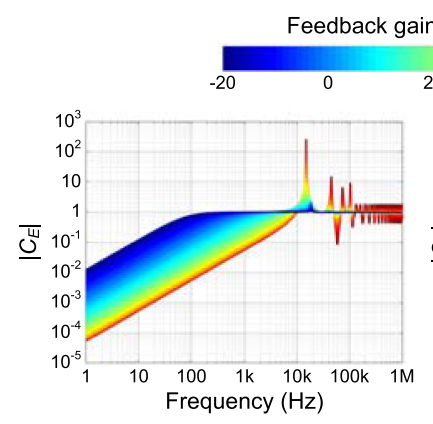

(a)

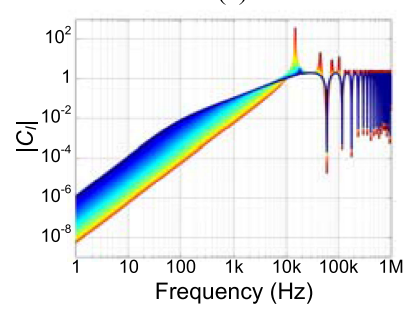

(c)

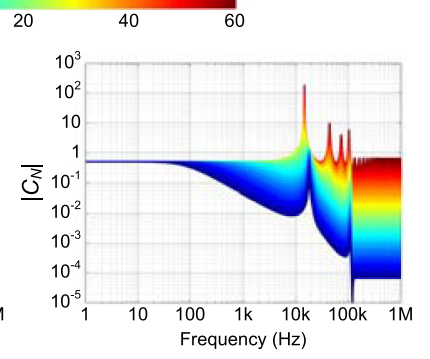

(b)

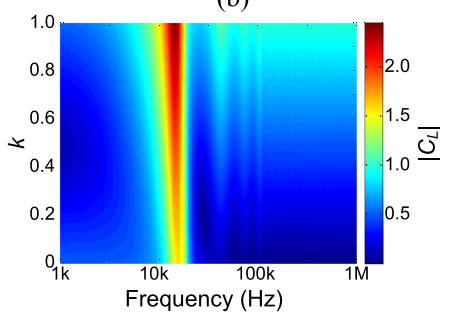

(d)
Fig. 16. (a)-(c) Coefficients for environmental noise, electronic noise and inherent jitter of the master laser for different feedback gains as indicated; (d) the coefficient for the link-induced jitter for different symmetry values $k$.

Based on this model, the out-of-loop timing jitter $J_{O L}$ is described by

$$
J_{O L}=C_{E} J_{E}+C_{N} J_{N}+C_{I} J_{I}+C_{L} H_{L R} J_{I}
$$

where

$$
\begin{aligned}
J_{N} & =-\frac{E_{B}}{H_{B}}+\frac{E_{P I}}{H_{B} G_{B} H_{P I}} \\
C_{E} & =\frac{1+H\left[1-\exp \left(-s T_{L}\right)\right]}{1+H\left[1+\exp \left(-2 s T_{L}\right)\right]} \\
C_{N} & =\frac{H \exp \left(-s T_{L}\right)}{1+H\left[1+\exp \left(-2 s T_{L}\right)\right]} \\
C_{I} & =\frac{1+H\left[1-\exp \left(-s T_{L}\right)\right]}{1+H\left[1+\exp \left(-2 s T_{L}\right)\right]}\left[\exp \left(-s T_{L}\right)-1\right] \\
C_{L} & =\frac{k+H\left[k-\exp \left(-2 s T_{L}\right)(1-k)\right]}{1+H\left[1+\exp \left(-2 s T_{L}\right)\right]} \exp \left(-s T_{L}\right) \\
H & =H_{B} G_{B} H_{P I} H_{P Z T} \\
k & =\frac{H_{L F} J_{I}}{H_{L R} J_{I}}
\end{aligned}
$$

As (8) indicates, $J_{O L}$ has 4 main contributions: the environmental noise imposed on the link, the electronic noise of the system, the master laser's inherent jitter and the link-induced jitter, with coefficients $C_{E}, C_{N}, C_{I}$ and $C_{L}$, respectively, where $k$ is a variable in the range $[0,1]$ that represents the degree of symmetry of link-induced jitter between the forward and backward link propagations.

The coefficients $C_{i}(i=E, N, I, L)$ for a 3.5 -km link can be calculated using the transfer functions of the experimental equipment. Fig. 16(a)-(c) show the calculated coefficients $\left|C_{E}\right|$, $\left|C_{N}\right|,\left|C_{I}\right|$ for different PI controller gain settings. High gain 


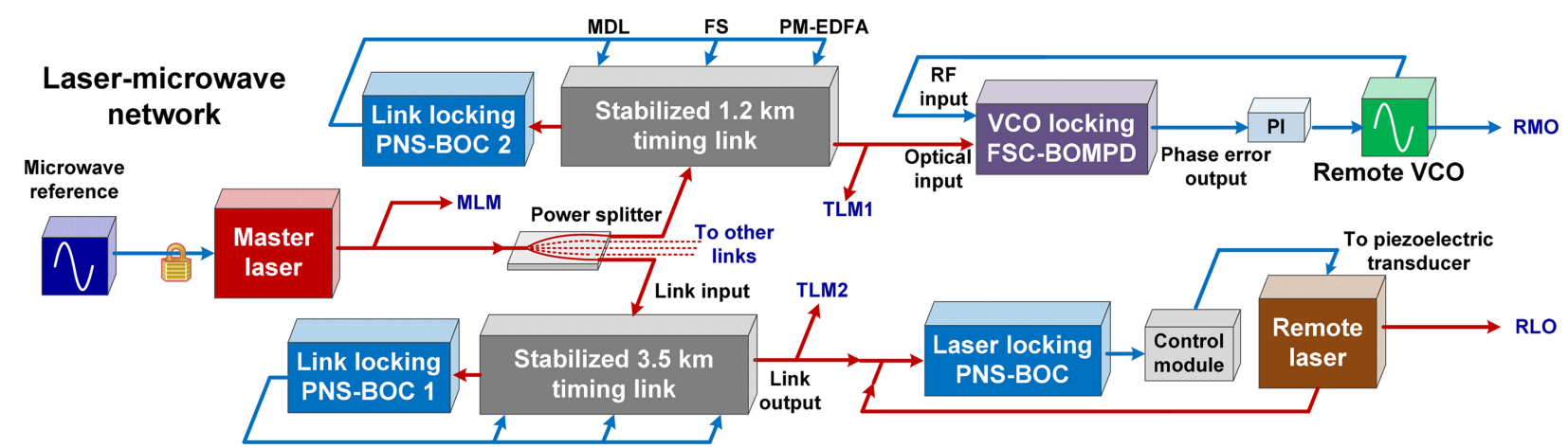

(a)
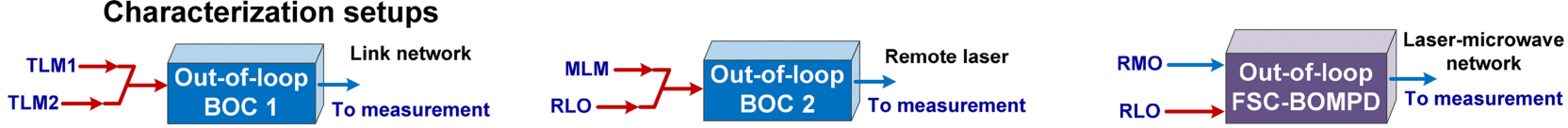

Free space optical path

(b)

Fig. 17. (a) Laser-microwave network (VCO, voltage-controlled oscillator); (b) Out-of-loop characterization setups [19].

is necessary to efficiently suppress the environmental noise below $1 \mathrm{kHz}$ (see Fig. 16(a)). However, the electronic noise from the BPD in the BOC and the PI controller rises with increasing gain (see Fig. 16(b)). Furthermore, large gain peaks appear in Fig. 16(a)-(c) at frequencies $n / 4 T_{L}$ (with odd integer $n$ ) as well as around the resonant frequency of the fiber stretcher (about $18 \mathrm{kHz}$ ), if the feedback gain is too high. Therefore, to optimize the system performance, a medium gain (e.g., $20 \mathrm{~dB}$ ) needs to be adopted. With $20 \mathrm{~dB}$ feedback gain setting, $\left|C_{I}\right|$ in Fig. 16(c) exponentially increases from 0.02 at $1 \mathrm{kHz}$ to 4.6 at $\sim 16 \mathrm{kHz}$, which means that the inherent jitter of the master laser can appear in the out-of-loop measurement through the feedback loop.

Also using a $20 \mathrm{~dB}$ gain value, $\left|C_{L}\right|$ for different symmetry values $k$ is calculated in Fig. 16(d). If the link-induced jitter from the forward and backward link transmission is almost identical, like in the case of the Gordon-Haus jitter in Fig. 13, then $k$ is about 0.5 , and $\left|C_{L}\right|$ is increased from 0.12 at $1 \mathrm{kHz}$ to 2 at $14.6 \mathrm{kHz}$ (see Fig. 16(d)). In the case of the nonlinearity-induced jitter shown in Fig. 14, if the backward link transmission power is much higher than for the forward path, the majority of the jitter is coming from the backward trip, $k$ is almost 0 . In Fig. $16(\mathrm{~d}),\left|C_{L}\right|$ is about 0.5 at $1 \mathrm{kHz}$ and approaches 1.5 at $16 \mathrm{kHz}$. On the other hand, if the forward power is greater than for the backward path, $k$ is close to 1 , and $\left|C_{L}\right|$ increases from 0.5 to 2.4 within $[1 \mathrm{kHz}, 14 \mathrm{kHz}]$. Above all, the link-induced jitter in Figs. 13 and 14 can transfer to the link output through the feedback loop, either partially, completely or even with amplification.

\section{LASER-MiCROWAVE NETWORK}

Employing all the techniques discussed in Sections II-IV, an attosecond-precision laser-microwave network [19], [65] is demonstrated using the setup shown in Fig. 17. The repetition rate of the master laser was locked to a microwave reference with a $10-\mathrm{Hz}$ feedback bandwidth to suppress long-term drifts. The timing signal from the master laser was distributed through a network that contains two independent fiber links of $1.2-\mathrm{km}$ and $3.5-\mathrm{km}$ length operated in parallel. The link outputs were used to synchronize a remote laser (e.g., serving as a pump laser at the FEL end station in Fig. 1) and a voltage-controlled oscillator (VCO) (e.g., serving as a microwave reference of the FEL linear accelerator in Fig. 1) simultaneously. PNS-BOCs were used for each locking loop and a feedback precision of $\sim 2$ as for the laser locking PNS-BOC is achieved with a low-noise BPD. The residual SOD and TOD of the links were compensated with additional dispersion-compensating fiber to suppress the linkinduced Gordon-Haus jitter and to minimize the output pulse durations for high signal-to-noise ratio (SNR) in the BOCs. The link power was adjusted to minimize the nonlinearity-induced jitter as well as to maximize the SNR for BOC locking. To eliminate power-fluctuation-induced drift, a feedback signal was sent to the EDFA to control its pump current (as shown in Fig. 10). The FSC-BOMPD, which was employed for opticalmicrowave locking, can provide a $>10 \times$ improvement for longterm timing stability compared with a fiber-coupled design.

Three characterization setups are adopted (see Fig. 17(b)): two timing link monitoring signals (TLM1 and 2) were sent to an out-of-loop BOC to evaluate the link network performance; the master laser monitoring signal (MLM) and the remote laser output signal (RLO) were sent to another BOC to characterize the remote laser synchronization; finally, the remote microwave output (RMO) and RLO were compared with an outof-loop FSC-BOMPD. The third setup is of great significance since it directly measures the true relative timing jitter between a remotely synchronized mode-locked laser and a microwave source, which has never been reported before.

The measurement results are shown in Figs. 18-21. Stabilization of the 4.7-km link network is operated continuously for 


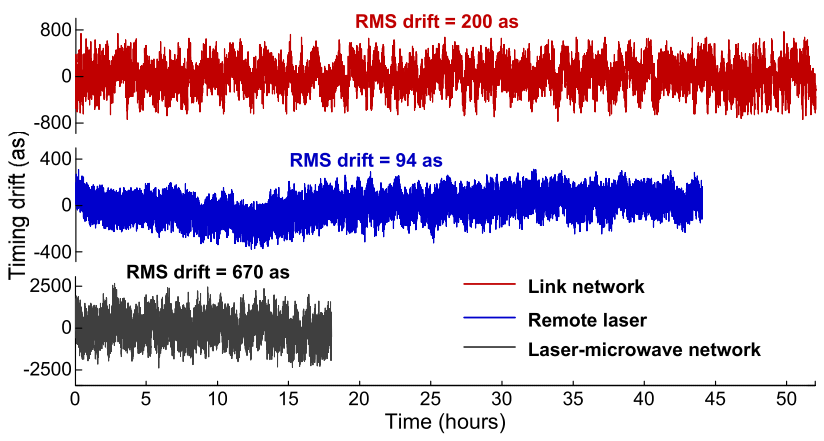

Fig. 18. Long-term timing drift results for the laser-microwave network [19].

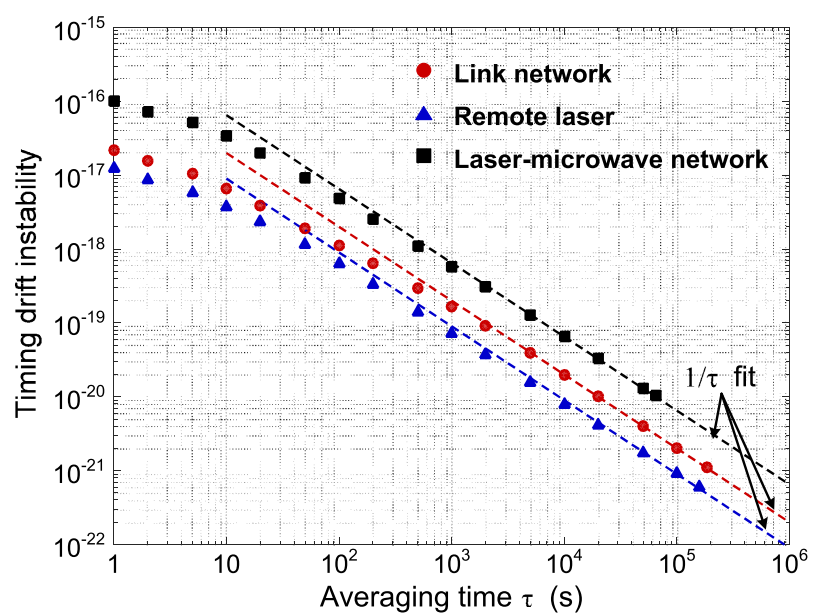

Fig. 19. Timing drift instability (Allan deviation) of the laser-microwave network versus averaging time $\tau$. Fits with $\tau^{-1}$ slope are shown [19].

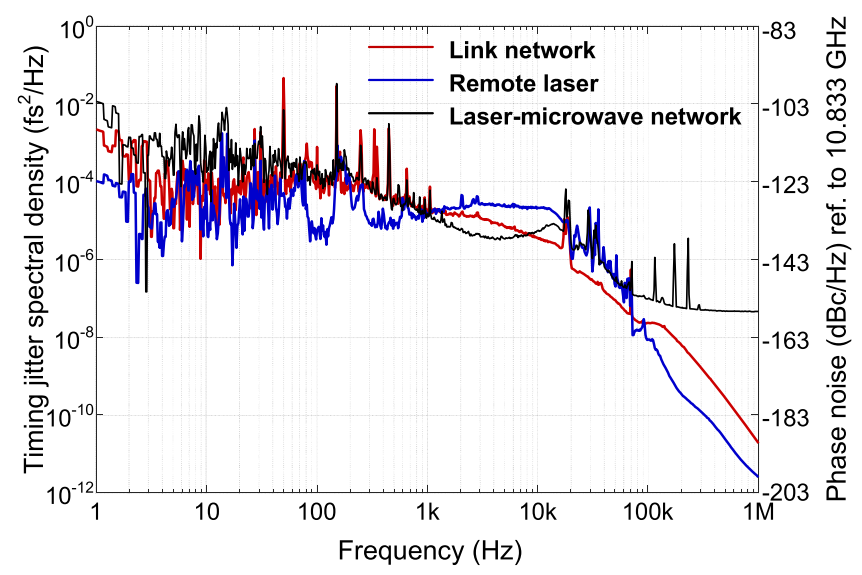

Fig. 20. Timing jitter spectral density of the laser-microwave network and the corresponding phase noise referenced to $10.833 \mathrm{GHz}$ within $[1 \mathrm{~Hz}, 1 \mathrm{MHz}]$ [19].

52 hours. The residual timing drift between TLM1 and 2 below $1 \mathrm{~Hz}$ amounts to only 200 as RMS (see Fig. 18, red curve). The relative timing drift instability is $2 \times 10^{-17}$ at 1 s averaging time $\tau$ and reduces to $2 \times 10^{-20}$ at $\tau=10^{4}$ s (see Fig. 19, red circles). The equivalent phase noise at $10.833 \mathrm{GHz}$ is lower than $-110 \mathrm{dBc} / \mathrm{Hz}$ at $1 \mathrm{~Hz}$ offset and drops below $-145 \mathrm{dBc} / \mathrm{Hz}$ above $20 \mathrm{kHz}$ (see Fig. 20, red curve); whereas the total inte-

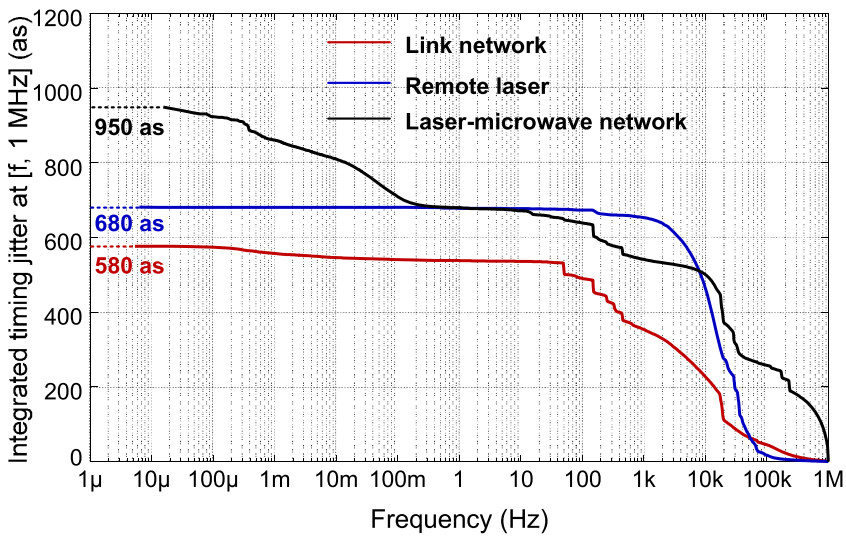

Fig. 21. Integrated timing jitter of three characterization setups in the lasermicrowave network [19].

grated timing jitter from $6 \mu \mathrm{Hz}$ to $1 \mathrm{MHz}$ is only 580 as (see Fig. 21, red curve).

Remote laser synchronization is achieved successfully for over 44 hours without interruption. The residual timing drift is less than 100 as RMS (see Fig. 18, blue curve), which is an order-of-magnitude improvement over previous results [45], and corresponds to a relative timing instability of $9 \times 10^{-22}$ at $10^{5} \mathrm{~s}$ (see Fig. 19, blue triangles). The integrated jitter is only 200 as in the range of $7 \mu \mathrm{Hz}-1 \mathrm{kHz}$ and 680 as for $7 \mu \mathrm{Hz}-$ $1 \mathrm{MHz}$ (see Fig. 21, blue curve).

Finally, the whole laser-microwave network shows an unprecedented long-term precision of 670 as RMS out-of-loop drift over 18 hours (see Fig. 18, black curve). Compared with previous frequency-comb-based microwave transfer results [66], this setup includes an additional fiber link and a remote laser synchronization system, yet it still achieves more than an orderof-magnitude improvement. The relative timing jitter between the two remotely synchronized devices within the full frequency range from $15 \mu \mathrm{Hz}$ to $1 \mathrm{MHz}$ is only 950 as RMS (see Fig. 21, black curve). To the best of our knowledge, this is the first attosecond-precision demonstration of remote optical-tomicrowave synchronization as well as the first demonstration of a synchronous laser-microwave network.

\section{CONCLUSION}

In summary, we have successfully achieved long-term-stable attosecond timing precision across a $4.7-\mathrm{km}$ fiber link network between remote optical and microwave devices. For shorter link lengths (e.g., $\sim 300 \mathrm{~m}$ ), because of lower required link operational power and smaller residual link dispersion, a better timing precision can be expected. Our technique can be easily transferred to multi-wavelength-laser networks [67], [68] with the help of two-color BOC [69], [70], and large-scale microwave networks with $\mu \mathrm{rad}$ precision [71]. The attosecond-precision laser-microwave network will enable next-generation FELs and other science facilities to operate with the unprecedented timing precision, thereby unfolding their full potential. This will drive new scientific efforts towards the making of atomic and molecular movies at the attosecond time scale, opening up many new research avenues in structural biology, drug development, 
chemistry, fundamental physics and material science. Besides, this technique will also accelerate developments in many other fields requiring the highest level of synchronization such as ultrastable clocks, gravitational-wave astronomy and coherent optical antenna arrays.

\section{REFERENCES}

[1] G. Dimitrakopoulos and P. Demestichas, "Intelligent transportation systems," IEEE Veh. Technol. Mag., vol. 5, no. 1, pp. 77-84, Mar. 2010.

[2] T. Ohanian and N. Phillips, Digital Filmmaking: The Changing Art and Craft of Making Motion Pictures, 2nd ed. Burlington, MA, USA: Focal Press, 2013, pp. 89-95.

[3] Photography-Hand-Held Cameras - Flash-Connector Dimensions, ISO Standard 519:1992, 1992.

[4] J. Laroche, "Estimating tempo, swing and beat locations in audio recordings," in Proc. IEEE Workshop Appl. Signal Process. Audio Acoust., 2001, pp. $135-138$.

[5] S. Yao, B. Mukherjee, and S. Dixit, "Advances in photonic packet switching: An overview," IEEE Commun. Mag., vol. 38, no. 2, pp. 84-94, Feb. 2000.

[6] P. J. Napier, A. R. Thompson, and R. D. Ekers, "The very large array: Design and performance of a modern synthesis radio telescope," Proc. IEEE, vol. 71, no. 11, pp. 1295-1320, Nov. 1983.

[7] J. F. Cliche and B. Shillue, "Precision timing control for radioastronomy: Maintaining femtosecond synchronization in the Atacama large millimeter array,” IEEE Control Syst., vol. 26, no. 1, pp. 19-26, Mar. 2006.

[8] D. R. DeBoer et al., "Australian SKA pathfinder: A high-dynamic range wide-field of view survey telescope," Proc. IEEE, vol. 97, no. 8, pp. 1507-1521, Aug. 2009.

[9] E. Middelberg and U. Bach, "High resolution radio astronomy using very long baseline interferometry," Rep. Prog. Phys., vol. 71, p. 066901, 2008.

[10] D. Dravins, T. Lagadec, and P. D. Nuñez, "Optical aperture synthesis with electronically connected telescopes," Nature Commun., vol. 6, 2015, Art. no. 6852.

[11] B. P. Abbott et al., "Observation of gravitational waves from a binary black hole merger," Phys. Rev. Lett., vol. 116, p. 061102, 2016.

[12] B. P. Abbott et al., "GW151226: Observation of gravitational waves from a 22-solar-mass binary black hole coalescence," Phys. Rev. Lett., vol. 116, p. 241103, 2016.

[13] P. W. Graham, J. M. Hogan, M. A. Kasevich, and S. Rajendran, "New method for gravitational wave detection with atomic sensors," Phys. Rev. Lett., vol. 110, p. 171102, 2013.

[14] S. Kolkowitz et al., "Gravitational wave detection with optical lattice atomic clocks," 2016, unpublished paper. [Online]. Available: https://arxiv.org/abs/1606.01859

[15] M. Altarelli et al., "XFEL: The European X-ray free-electron laser," DESY, Hamburg, Germany, Tech. Des. Rep., 2006-097, 2006.

[16] E. Allaria et al., "Highly coherent and stable pulses from the FERMI seeded free-electron laser in the extreme ultraviolet," Nature Photon., vol. 6, pp. 699-704, 2012.

[17] P. Emma et al., "First lasing and operation of an Ångstrom-wavelength free-electron laser," Nature Photon., vol. 4, pp. 641-647, 2010.

[18] J. Stohr, "Linac coherent light source II (LCLS-II) conceptual design report," SLAC, Menlo Park, CA, USA, Des. Rep. SLAC-R-978, 2011.

[19] M. Xin et al., "Attosecond precision multi-kilometer laser-microwave network," Light: Science \& Applications 5, e16187, 2017.

[20] E. Prat and S. Reiche, "Simple method to generate terawatt-attosecond X-ray free-electron-laser pulses," Phys. Rev. Lett., vol. 114, p. 244801, 2015.

[21] C. Kupitz et al., "Serial time-resolved crystallography of photosystem II using a femtosecond X-ray laser," Nature, vol. 513, pp. 261-265, 2014.

[22] H. Öström et al., "Probing the transition state region in catalytic CO oxidation on Ru," Science, vol. 347, pp. 978-982, 2015.

[23] F. Calegari et al., "Ultrafast electron dynamics in phenylalanine initiated by attosecond pulses," Science, vol. 346, pp. 336-339, 2014.

[24] S. K. Son, L. Young, and R. Santra, "Impact of hollow-atom formation on coherent X-ray scattering at high intensity," Phys. Rev. A., vol. 83, p. 033402, 2011.

[25] S. P. Hau-Riege, "Photoelectron dynamics in X-ray free-electron-laser diffractive imaging of biological samples," Phys. Rev. Lett., vol. 108, p. 238101, 2012.
[26] C. W. Chou, D. B. Hume, T. Rosenband, and D. J. Wineland, "Optical clocks and relativity," Science, vol. 329, pp. 1630-1633, 2010.

[27] T. L. Nicholson et al., "Systematic evaluation of an atomic clock at $2 \times$ $10^{-18}$ total uncertainty," Nature Commun., vol. 6, 2015, Art. no. 6896.

[28] D. Meiser, J. Ye, D. R. Carlson, and M. J. Holland, "Prospects for a millihertz-linewidth laser," Phys. Rev. Lett., vol. 102, p. 163601, 2009.

[29] C. Hagemann et al., "Ultrastable laser with average fractional frequency drift rate below $5 \times 10^{-19} / \mathrm{s}$," Opt. Lett., vol. 39, pp. 5102-5105, 2014 .

[30] T. K. Kim et al., "Sub-100-as timing jitter optical pulse trains from modelocked Er-fiber lasers," Opt. Lett., vol. 36, pp. 4443-4445, 2011.

[31] A. J. Benedick, J. G. Fujimoto, and F. X. Kärtner, "Optical flywheels with attosecond jitter," Nature Photon., vol. 6, pp. 97-100, 2012.

[32] T. R. Schibli et al., "Attosecond active synchronization of passively mode-locked lasers by balanced cross correlation," Opt. Lett., vol. 28 , pp. 947-949, 2003.

[33] J. Kim et al., "Long-term femtosecond timing link stabilization using a single-crystal balanced cross correlator," Opt. Lett., vol. 32, pp. 1044-1046, 2007.

[34] J. Kim, F. X. Kärtner, and F. Ludwig, "Balanced optical-microwave phase detectors for optoelectronic phase-locked loops," Opt. Lett., vol. 31, pp. 3659-3661, 2006.

[35] M. Y. Peng, A. Kalaydzhyan, and F. X. Kärtner, "Balanced opticalmicrowave phase detector for sub-femtosecond optical-RF synchronization," Opt. Exp., vol. 22, pp. 27102-27111, 2014.

[36] S. Foreman et al., "Coherent optical phase transfer over a 32-km fiber with $1 \mathrm{~s}$ instability at $10^{-17}$," Phys. Rev. Lett., vol. 99, p. 153601, 2007.

[37] I. Coddington et al., "Coherent optical link over hundreds of metres and hundreds of terahertz with subfemtosecond timing jitter," Nature Photon., vol. 1, pp. 283-287, 2007.

[38] J. Kim, J. A. Cox, J. Chen, and F. X. Kärtner, "Drift-free femtosecond timing synchronization of remote optical and microwave sources," Nature Photon., vol. 2, pp. 733-736, 2008.

[39] O. Lopez, A. Amy-Klein, M. Lours, C. Chardonnet, and G. Santarelli, "High-resolution microwave frequency dissemination on an 86-km urban optical link," Appl. Phys. B, vol. 98, pp. 723-727, 2010.

[40] K. Predehl et al., "A 920-kilometer optical fiber link for frequency metrology at the 19th decimal place," Science, vol. 336, pp. 441-444, 2012

[41] S. Schulz et al., "Femtosecond all-optical synchronization of an X-ray free-electron laser," Nature Commun., vol. 6, 2015, Art. no. 5938.

[42] F. R. Giorgetta et al., "Optical two-way time and frequency transfer over free space," Nature Photon., vol. 7, pp. 434-438, 2013.

[43] J. D. Deschênes et al., "Synchronization of distant optical clocks at the femtosecond level," Phys. Rev. X, vol. 6, p. 021016, 2016.

[44] M. Y. Peng et al., "Long-term stable, sub-femtosecond timing distribution via a 1.2-km polarization-maintaining fiber link: Approaching $10^{-21}$ link stability," Opt. Exp., vol. 21, pp. 19982-19989, 2013.

[45] M. Xin et al., "One-femtosecond, long-term stable remote laser synchronization over a 3.5-km fiber link," Opt. Exp., vol. 22, pp. 14904-14912, 2014.

[46] K. Safak et al., "All fiber-coupled, long-term stable timing distribution for free electron lasers with few-femtosecond jitter," Struct. Dyn., vol. 2, pp. 041715-1-041715-10, 2015.

[47] R. Wilcox, J. M. Byrd, L. Doolittle, G. Huang, and J. W. Staples, "Stable transmission of radio frequency signals on fiber links using interferometric delay sensing," Opt. Lett., vol. 34, pp. 3050-3052, 2009.

[48] J. M. Glownia et al., "Time-resolved pump-probe experiments at the LCLS," Opt. Exp., vol. 18, pp. 17620-17630, 2010.

[49] R. P. Scott, C. Langrock, and B. H. Kolner, "High-dynamic-range laser amplitude and phase noise measurement techniques," IEEE J. Sel. Topics Quantum Electron., vol. 7, no. 4, pp. 641-655, Jul./Aug. 2001.

[50] F. Quinlan et al., "Exploiting shot noise correlations in the photodetection of ultrashort optical pulse trains," Nature Photon., vol. 7, pp. 290-293, 2013.

[51] W. Sun et al., "Broadband noise limit in the photodetection of ultralow jitter optical pulses," Phys. Rev. Lett., vol. 113, p. 203901, 2014.

[52] J. Kim, J. Chen, J. Cox, and F. X. Kärtner, "Attosecond-resolution timing jitter characterization of free-running mode-locked lasers," Opt. Lett., vol. 32, pp. 3519-3521, 2007.

[53] J. A. Cox, A. H. Nejadmalayeri, J. Kim, and F. X. Kärtner, "Complete characterization of quantum-limited timing jitter in passively mode-locked fiber lasers," Opt. Lett., vol. 35, pp. 3522-3524, 2010.

[54] Y. Song, C. Kim, K. Jung, H. Kim, and J. Kim, "Timing jitter optimization of mode-locked Yb-fiber lasers toward the attosecond regime," Opt. Exp., vol. 19, pp. 14518-14525, 2011

[55] K. Şafak et al., "Jitter analysis of timing-distribution and remote-laser synchronization systems," Opt. Exp., vol. 24, pp. 21752-21766, 2016. 
[56] A. H. Nejadmalayeri, F. N. C. Wong, T. D. Roberts, P. Battle, and F. X. Kärtner, "Guided wave optics in periodically poled KTP: Quadratic nonlinearity and prospects for attosecond jitter characterization," Opt. Lett., vol. 34, pp. 2522-2524, 2009.

[57] P. T. Callahan, K. Şafak, P. Battle, T. D. Roberts, and F. X. Kärtner, "Fibercoupled balanced optical cross-correlator using PPKTP waveguides," Opt. Exp., vol. 22, pp. 9749-9758, 2014.

[58] A. Kalaydzhyan et al., "Optical-to-microwave synchronization with subfemtosecond daily drift," presented at the 30th Eur. Freq. Time Forum, York, U.K., 2016.

[59] K. Jung and J. Kim, "Sub-femtosecond synchronization of microwave oscillators with mode-locked Er-fiber lasers," Opt. Lett., vol. 37, pp. 2958-2960, 2012.

[60] M. Lessing, H. S. Margolis, C. T. A. Brown, P. Gill, and G. Marra "Suppression of amplitude-to-phase noise conversion in balanced opticalmicrowave phase detectors," Opt. Exp., vol. 21, pp. 27057-27062, 2013.

[61] G. P. Agrawal, Nonlinear Fiber Optics, 4th ed. Burlington, MA, USA Academic, 2013.

[62] J. C. Diels and W. Rudolph, Ultrashort Laser Pulse Phenomena, 2nd ed. Burlington, MA, USA: Academic, 2006.

[63] J. Hult, "A fourth-order Runge-Kutta in the interaction picture method for simulating supercontinuum generation in optical fibers," IEEE J. Lightw. Technol., vol. 25, no. 12, pp. 3770-3775, Dec. 2007.

[64] J. P. Gordon and H. A. Haus, "Random walk of coherently amplified solitons in optical fiber transmission," Opt. Lett., vol. 11, pp. 665-667, 1986.

[65] M. Xin et al., "Attosecond precision multi-km laser-microwave network," presented at the Conf. Lasers Electro-Opt., San Jose, CA, USA, 2016, Paper JTh4C.6.

[66] K. Jung et al., "Frequency comb-based microwave transfer over fiber with $7 \times 10^{-19}$ instability using fiber-loop optical-microwave phase detectors," Opt. Lett., vol. 39, pp. 1577-1580, 2014.

[67] K. Şafak et al., "Synchronous mode-locked laser network with sub-fs jitter and multi-km distance," presented at the Conf. Lasers Electro-Opt., San Jose, CA, USA, 2016, Paper SM4H.2.

[68] K. Şafak et al., "Synchronous mode-locked laser network with $20^{\text {th }}$ decimal timing precision," to be published.

[69] H. Li et al., "Remote two-color optical-to-optical synchronization between two passively mode-locked lasers," Opt. Lett., vol. 39, pp. 53255328,2014

[70] G. Zhou, M. Xin, F. X. Kärtner, and G. Chang, "Timing jitter of Raman soliton," Opt. Lett., vol. 40, pp. 5105-5108, 2015.

[71] W. Wang et al., "High precision synchronization of a large-scale microwave network over stabilized fiber links," presented at the Conf. Lasers Electro-Opt., San Jose, CA, USA, 2016, Paper SM4H.5.

Ming Xin (M'12) received the bachelor's and doctor's degrees in electronic engineering from Tsinghua University, Beijing, China, in 2005 and 2010, respectively. From 2010 to 2011, he was a Project Manager with China Mobile Research Institute of China Mobile, Beijing. In July 2011, he joined the CFEL Ultrafast Optics and X-rays Group of DESY, Hamburg, Germany, as a Team Leader. Since 2015, he has also been a Scientist with the Research Laboratory of Electronics, Massachusetts Institute of Technology, Cambridge, MA, USA His research interests include network contention resolution, optical communication, attosecond photonics, noise theory of mode-locked lasers, timing distribution systems, and silicon photonics.

Kemal Şafak received the B.S. degree from Middle East Technical University, Ankara, Turkey, in 2009, and the M.S. degree from Friedrich Schiller University, Jena, Germany, in 2012. Since 2012, he has been working toward the Ph.D. degree with Deutsches Elektronen-Synchrotron, Hamburg, Germany. From 2010 to 2012, he was a Research Assistant with the Fraunhofer Institute for Applied Optics and Precision Engineering, Jena. His current research interests include laser-based time and frequency transfer and low-noise mode-locked lasers and their applications in metrology.

Michael Y. Peng received the B.S. degree from the University of California, Berkeley, CA, USA, in 2009, and the S.M. and Ph.D. degrees in electrical engineering and computer science from Massachusetts Institute of Technology, Cambridge, MA, USA, in 2011 and 2015, respectively. Since September 2015, he has been an Optical Engineer with the Optical Communications Group, Jet Propulsion Laboratory, Pasadena, CA. His research interests include optical communications, microwave photonics, and optical frequency combs for highprecision metrology.
Aram Kalaydzhyan started studying physics at the Lomonosov Moscow State University, Moskva, Russia, in 2007. In 2010, he continued his education and received the B.Sc. and M.Sc. degrees from the University of Hamburg, Hamburg, Germany, in 2013 and 2015, respectively. He is currently working toward the $\mathrm{Ph} . \mathrm{D}$. degree in physics at CFEL, Hamburg. During the period of studies in Hamburg, he was a Research Assistant with HASYLAB and CFEL at DESY. His current research interests include ultrastable microwave extraction from femtosecond laser frequency combs, FPGA-based data acquisition, and control systems.

Patrick T. Callahan (M'08) received the B.A. degree in physics from Cornell University, Ithaca, NY, USA, in 2008, and the M.S. degree in electrical engineering from Johns Hopkins University, Baltimore, MD, USA, in 2010. He is currently working toward the Ph.D. degree in electrical engineering at the Massachusetts Institute of Technology, Cambridge, MA, USA. From 2008 to 2011, he was a Member of the Professional Staff with the Johns Hopkins University Applied Physics Laboratory, Laurel, MD, USA. His current research interests include low-phase noise photonic oscillators, mode-locked lasers, microwave photonics, and integrated photonics.

Wenting Wang received the M.S. degree in optical engineering from Nankai University, Tianjin, China, in 2013. He is currently working toward the Ph.D. degree at the Institute of Semiconductors, Chinese Academy of Sciences, Beijing, China, and Deutsches Elektronen-Synchrotron, Hamburg, Germany. His research interests include ultralow-noise microwave/terahertz generation, optical signal processing, integrated microwave photonics, and high-precision time and frequency transfer.

Oliver D. Mücke received the Diploma and Ph.D. degrees in physics from Universität Karlsruhe, Karlsruhe, Germany, in 1999 and 2003, respectively. From 2003 to 2007, he was a Feodor Lynen Fellow of the Alexander von Humboldt Foundation and later a Postdoctoral Associate with the MIT Research Laboratory of Electronics. In 2007, he joined the Photonics Institute, TU Vienna, Austria, supported by a FWF Lise Meitner Senior Postdoc Fellowship (09/2008-09/2010). Since July 2011, he has been a Senior Scientist with DESY CFEL, Hamburg, Germany. His research interests include CEP-stable few-cycle pulse generation from octave-spanning Ti:sapphire oscillators and parametric amplifiers, high-energy multioctave waveform synthesis, strong-field physics and attoscience, highly efficient intense terahertz-pulse generation, laser-driven sources of particles and hard-X-rays, frequency-comb technology for optical clocks, extreme nonlinear optics in solids and nanostructures, and lightwavedriven petahertz electronics.

Franz X. Kärtner (F'09) Heads the Ultrafast Optics and X-Rays Group, DESY CFEL, Hamburg, Germany, and is a Professor of physics with the University of Hamburg, Hamburg, and an Adjunct Professor of electrical engineering with the Massachusetts Institute of Technology, Cambridge, MA, USA. His research interests include noise in electronic and optical sources, ultrashort pulse lasers, high-energy subcycle waveform synthesis, frequency combs and precision timing in advanced accelerators and light sources, attosecond physics, and compact free-electron lasers. 